\title{
Fast Level Set Evolution of Region Based Segmentation of Satellite and Medical Imagery on Noisy Images
}

\author{
G. Raghotham Reddy, K. Ramudu, A. Srinivas, and R. Rameshwar Rao
}

\begin{abstract}
-in this paper, we proposed a novel global segmentation method for satellite images with active contour model on noisy images with ten percentage of salt and pepper. It was implemented with a special technique selective binary and Gaussian filtering regularized level set evolution. First we selectively penalize the level set function to be binary and then use a Gaussian smoothing kernel to regularize it. The advantages of our method is a new region based signed pressure force(SPF) function is proposed, which can step effectively the contour at weak or blurred edges and automatically detect the interior and exterior boundaries with the initial contour being any where in the images effected with noise. The proposed method can implement by the simple finite difference scheme. Experiments on satellite images with noise demonstrate the advantages of the proposed method over the Chan-Vase (CV) active contour in terms of the number of Iterations.
\end{abstract}

Index Terms-Active contours, Image segmentation, Chanvase model, Level set method.

\section{INTRODUCTION}

Image segmentation is a fundamental problem in image processing and computer vision. Extensive study has been made and many techniques have been proposed [1], among which the ACM $[2,3]$ is one of the most successful methods The basic idea of ACM is to evolve a curve under some constraints to extract the desired object. According to the nature of constraints, the existing ACMs can be categorized into two types: edge-based models $[4,5]$ and region-based models [6, 7-10].

Image segmentation plays an important role in the field of image understanding, image analysis and pattern identification. The foremost essential goal of the segmentation process is to partition an image into regions that are homogeneous (uniform) with respect to one or more self characteristics and features. Active contour methods are applied in a wide range of problems including visual tracking and image segmentation [11]-[14]. The basic idea is to allow a contour to deform so as to minimize a given energy functional in order to produce the desired segmentation. Two main categories exist for active contours: edge-based and region-based. Edge-based active contour models utilize image gradients in order to identify object boundaries, e.g., [15], [16]. Region- based ACMs have many advantages over edge-based ones. Initially, region-

Manuscript received May 13, 20111; revised June 19, 2011.

G.Raghotham Reddy, is with the Department of ECE, KITS, Warangal15, India.(grr_ece@yahoo.com).

K.Ramudu, is with the Department of ECE, KITS, Warangal-15, India. (ramudukama@gmail.com).

A.Srinivas, is with the Department of ECE, KITS, Warangal-15, India. (srinivas_azmeera@yahoo.com).

R.Rameshwar Rao, is with the Department of ECE, OUCE, OU, Hyderabad(rameshwarrao@hotmail.com). based models utilize the statistical information inside and outside the contour to control the evolution, which are less sensitive to noise; give better performance for images with weak edges or without edges and they are significantly less sensitive to the location of initial contour, further more they can efficiently detect the exterior and interior boundaries simultaneously.

One of the most popular region-based models is the $\mathrm{C}-\mathrm{V}$ model [3], which is based on Mumford-Shah segmentation techniques [7] and has been successfully applied to binary phase segmentation. As pointed in [3], the $\mathrm{C}-\mathrm{V}$ model can automatically detect all of the contours, no matter where the initial contour starts in the image. So we can say that the C$\mathrm{V}$ model has the global segmentation property to segment all objects in an image in comparison with the GAC model.

In this paper, we propose a new region-based ACM, which shares the advantage of the $\mathrm{C}-\mathrm{V}$. We utilize the statistical information inside and outside the contour to construct a region-based signed pressure force (SPF) function [17], which is able to control the direction of evolution. The proposed SPF function has opposite signs around the object boundary, so the contour can shrink when it is outside the object or expand when inside the object.

In this paper we do propose a novel level set method, i.e. Selective Binary and Gaussian Filtering Regularized Level Set Evolution (SBGFRLS), to implement our model. Unlike in Traditional Level Set methods, this method avoids the calculation of SDF and costly re-initialization [18].Firstly the Level Set Evolution will be penalized by using a selective step and then we use a Gaussian filter to regularize it. The Gaussian filter can make the level set function smooth and the evolution more stable. It is worth noting that our proposed method is general and robust, and it can be applied to classical ACMs, C-V model [3], PS model [8, 19], and LBF model [20, 21]. Furthermore, computational complexity analysis shows that the SBGFRLS method is more efficient than the traditional level set methods. In addition, the proposed model implemented with SBGFRLS has a property of selective global segmentation, which can not only extract the desired objects, but also accurately extract all the objects with interior and exterior boundaries. This paper is organized as follows: $\mathrm{C}-\mathrm{V}$ models. In section 3 we describe our method and show how to construct the region-based SPF function.

The advantages of our model over $\mathrm{C}-\mathrm{V}$ models are also discussed. Section 4 validates our method by extensive experiments on satellite images with adding salt and pepper noise

\section{The Chan-Vese (CV) Model}

Chan and Vese [3] proposed an ACM which can be seen as a special case of the Munford-Shah problem [7]. For a given image $\mathrm{I}$ in domain $\mathrm{X}$, the $\mathrm{C}-\mathrm{V}$ model is formulated by 
minimizing the following energy functional:

$$
E^{c v}=\lambda_{1} \int_{\text {inside }(c)}\left|I(x)-C_{1}\right|^{2} d x+\lambda_{2} \int_{\text {outside }(c)}\left|I(x)-C_{2}\right|^{2} d x, x \varepsilon \Omega
$$

where $\mathrm{C}_{1}$ and $\mathrm{C}_{2}$ are two constants which are the average intensities inside and outside the contour, respectively. With the level set method, we assume

$$
\begin{aligned}
C & =\{x \in \Omega: \varphi(x)=0\} \\
\text { Inside }(C) & =\{x \in \Omega: \varphi(x)>0\} \\
\text { Outside }(C) & =\{x \in \Omega: \varphi(x)<0\}
\end{aligned}
$$

By minimizing esq.: (1), we solve $\mathrm{C} 1$ and $\mathrm{C} 2$ as follows

$$
\begin{array}{r}
C_{1}=\frac{\int_{\Omega} I(x) \cdot H(\phi) d x}{\int_{\Omega} H(\phi) d x} \\
C_{2}=\frac{\int_{\Omega} I(x) \cdot(1-H(\phi)) d x}{\int_{\Omega}(1-H(\phi)) d x}
\end{array}
$$

By incorporating the length and area energy terms into Eq. (1) and minimizing them, we obtain the corresponding variational level set formulation as follows:

$$
\frac{\partial \phi}{\partial t}=\partial(\phi)\left[\mu \nabla\left(\frac{\nabla \phi}{|\nabla \phi|}-v-\lambda_{1}\left(I-C_{1}\right)^{2}+\lambda_{2}\left(I-C_{2}\right)^{2}\right)\right]
$$

where $\mu \geq 0 v \geqslant 0, \lambda_{1}>0, \lambda_{2}>0$ are fixed parameters, $\mu$ controls the smoothness of zero level set, $v$ increases the propagation speed, $\lambda_{1}$ and $\lambda_{2}$ control the image data driven force inside and outside the contour, respectively. $\nabla$ Is the gradient operator. $H(\phi)$ Is the Heaviside function and $\delta(\phi)$ is the Dirac function. Generally, the regularized versions are selected as follows:

$$
\begin{aligned}
& H_{\varepsilon}(z)=\frac{1}{2}\left(1+\frac{2}{\pi} \arctan \left(\frac{z}{\varepsilon}\right)\right), \\
& \delta_{\varepsilon}(z)=\frac{1}{\pi} \cdot \frac{\varepsilon}{\varepsilon^{2}+z^{2}}, Z \varepsilon R
\end{aligned}
$$

\section{THE PROPOSED MODEL}

\section{A. The Design of SPF Function}

The SPF function defined in [16] has values in the range $[-1,1]$. It changes the signs of the pressure forces inside and outside the region of interest so that the contour shrinks when outside the object, or expands when inside the object. Based on the analysis in Section 2, we construct the SPF function as follows:

$$
\operatorname{spf}(I(x))=\frac{I(x)-\frac{c_{1}+c_{2}}{2}}{\max \left(\left|I(x)-\frac{c_{1}+c_{2}}{2}\right|\right)}, x \varepsilon R
$$

where $\mathrm{C}_{1}$ and $\mathrm{C}_{2}$ are defined in Eq. (2) and (3), respectively.

The significance of Eq. (6) is that, we assume the intensities inside and outside the object are homogeneous. It is intuitive that $\min (I(x)) \leq c_{1}, c_{2} \leq \max (I(x))$, and the equal signs cannot be obtained simultaneously wherever the contour is. Hence, there is

$$
\min (I(x)) \leq \frac{c_{1}+c_{2}}{2} \leq \max (I(x)), x \varepsilon \Omega
$$

Obviously, the signs of the SPF function in Eq. (6) are identical; it can serve as an SPF function. Substituting the
SPF function in Eq. (6) for GAC model, the level set formulation of the proposed model is as follows:

$$
\frac{\partial \phi}{\partial t}=\operatorname{spf}(I(x)) \cdot\left(\operatorname{div}\left(\frac{\nabla \phi}{|\nabla \phi|}\right)+\alpha\right)|\nabla \phi|+\nabla \operatorname{spf}(I(x)) . \nabla \phi, x \varepsilon R
$$

\section{B. Implementation}

In the traditional level set methods, the level set function is initialized to be an SDF to its interface in order to prevent it from being too steep or flat near its interface, and reinitialization is required in the evolution. The undesirable side effect in many existing re-initialization methods is moving the zero level set away from its interface. Furthermore, it is difficult to decide when and how to apply the re-initialization. In addition, re-initialization is a very expensive operation. To solve these problems, we propose a novel level set method, which utilizes a Gaussian filter to regularize the selective binary level set function after each iteration. The procedure of penalizing level set function to be binary is optional according to the desired property of evolution. If we want local segmentation property, the procedure is necessary; otherwise, it is unnecessary.

In our method, the level set function can be initialized to constants, which have different signs inside and outside the contour. This is very simple to implement in practice. In the traditional level set methods, the curvature-based term $\operatorname{div}(\nabla \phi /|\nabla \phi|)|\nabla \phi|$ is usually used to regularize the level set function $\varphi$. Since $\varphi$ is an $\operatorname{SDF}$ that satisfies $|\nabla \phi|-1$ [17], the regularized term can be rewritten as $\Delta \phi$, which is the Laplacian of the level set function $\varphi$. As pointed out in [21] and based on the theory of scale-space [22], the evolution of a function with its Laplacian is equivalent to a Gaussian kernel filtering the initial condition of the function. Thus we can use a Gaussian filtering process to further regularize the level set function The standard deviation of the Gaussian filter can control the regularization strength, just as the parameter $\mu$ in Eq. (4) does. Since we utilize a Gaussian filter to smooth the level set function to keep the interface regular, the regular term $\operatorname{div}(\nabla \phi /|\nabla \phi|)|\nabla \phi|$ is unnecessary. In addition, the term $\nabla s p f . \nabla \phi$ in Eq. (8) can also be removed, because our model utilizes the statistical information of regions, which has a larger capture range and capacity of anti-edge leakage. Finally, the level set formulation of the proposed model can be written as follows:

$$
\frac{\partial \phi}{\partial t}=\operatorname{spf}(I(x)) . \alpha|\nabla \phi|, x \boldsymbol{\Omega} \Omega
$$

The main procedures of the proposed algorithm are summarized as follows:

1) Initialize the level set function $\Phi$ as

$$
\phi(x, t=0)=\left\{\begin{array}{cc}
-\rho x \varepsilon \Omega_{0}-\delta \Omega_{0} \\
0 & x \varepsilon \delta \Omega_{0} \\
\rho & x \varepsilon \Omega-\Omega_{0}
\end{array}\right.
$$

where $\rho>0$ is a constant, $\Omega_{0}$ is a subset in the image domain $\Omega$ and is the boundary of $\delta \Omega_{0}$ is the boundary of $\Omega_{0}$.

2) Compute $c_{1}(\phi) a n d c_{2}(\phi)$ using Eqs. (2) and (3), 
3) Evolve the level set function according to Eq. (9).

4) Let $\phi-1$ if $\phi>0$; otherwise, $\phi=-1$;

This step has the local segmentation property. If we want to selectively segment the desired objects, this step is necessary; otherwise, it is unnecessary.

5) Regularize the level set function with a Gaussian filter, i.e. $\phi=\phi^{*} G_{\sigma}$

6) Check whether the evolution of the level set function has converged. If not, return to step 2.

\section{Compare our proposed model over CV model}

Compared with the $\mathrm{C}-\mathrm{V}$ model, our model can extract objects whose boundaries are distinctive while interior intensities are not homogeneous (see Fig. 1 and 2 for example). Moreover, our model can selectively extract the desired object by setting the initial contour intersecting or surrounding the desired boundaries, while the $\mathrm{C}-\mathrm{V}$ model will extract all the objects. In addition, the evolution direction in our model can be controlled to obtain satisfying segmentation results, while the $\mathrm{C}-\mathrm{V}$ model may get disordered results. Further more, our model can extract all the objects with the initial contour being set anywhere, while the $\mathrm{C}-\mathrm{V}$ model may be trapped into the local minima and then result in unsatisfied segmentation. The comparison of experimental results between $\mathrm{C}-\mathrm{V}$ model and our model on satellite images with salt and pepper noise indicate that computational complexity decreases as shown in figure below.

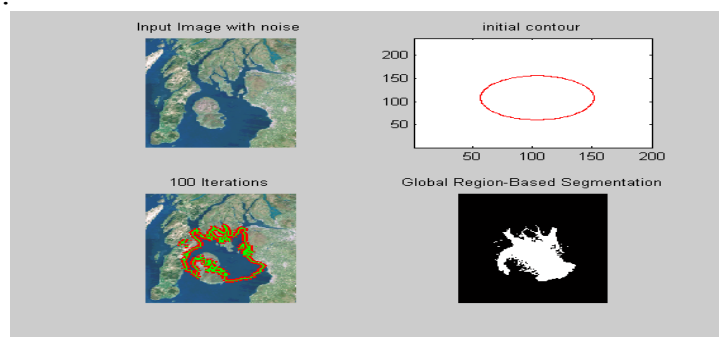

Figure (1) shows the results of (a) input image with noise (b) initial contour (c) active contour segmentation with 100 iterations (d) global region based segmentation.

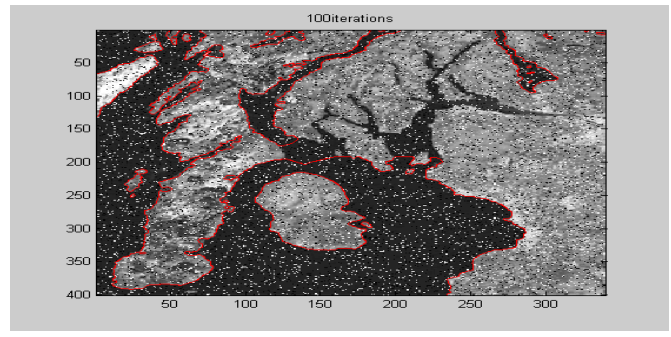

Figure (2) shows the final fast global perfect segmentation with proposed model with iterations 100 with same image in fig1.

\section{THE NOISE MODEL}

In this section we focus our attention on the noise model. The chosen descriptor for this port is

$$
f_{n}(x)=F(p(y(x)))
$$

where $\mathrm{p}$ is the pdf of some image features $\mathrm{y}(\mathrm{x})$ and $\mathrm{F}$ is at least C. In our study, we consider that $p$ belongs to the exponential family .This family is comprehensive enough to cover noise models in most image acquisition systems encountered in practice e.g. Gaussian, exponential, Poisson, Raleigh to cite a few.

\section{EXPERIMENTAL RESULTS}

The segmentation of image takes an important branch in the surgery navigation and tumor radiotherapy. However, duly in this project we considered satellite based images with salt and pepper noise added to the text images. Segmentation was done on the images globally for both the models with active contours and level set evolution on noisy images with number of iterations as shown in figures (3) and figure (4).Our method has best globally segmented regions with satellite noisy images over the CV model with active contours and level set methods.

We are testing on other particular images like noisy (salt $\&$ pepper) satellite and medical images for various number of iterations and the results are as shown below.
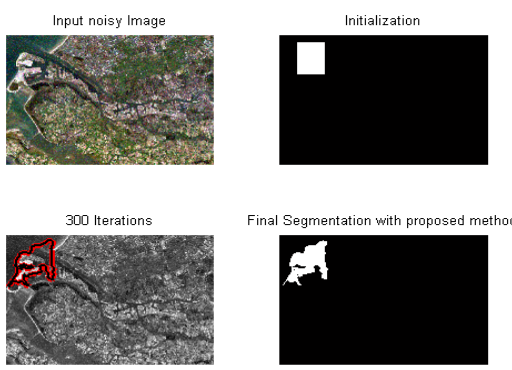

Figure (3a) The CV model with 300 iterations

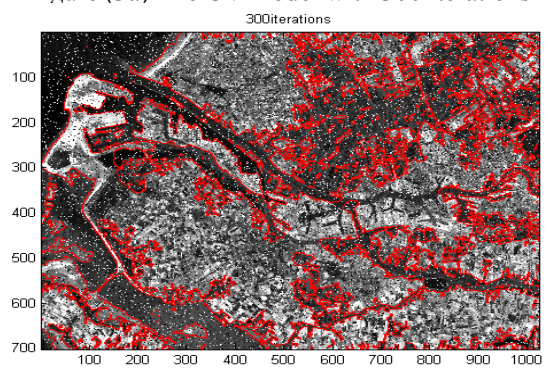

Figure (3b) the proposed model with 100 iterations.

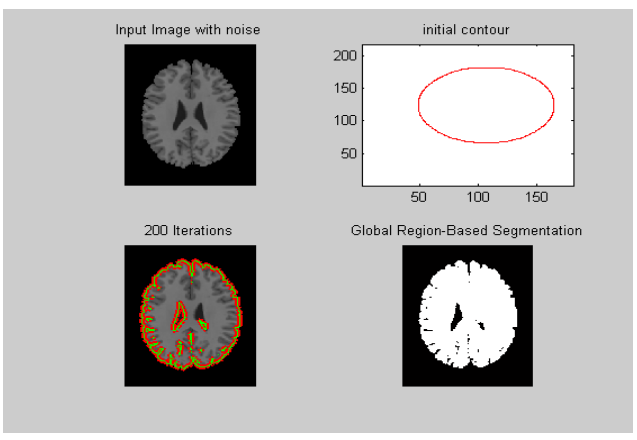

Figure (4a) The CV model with 200 iterations

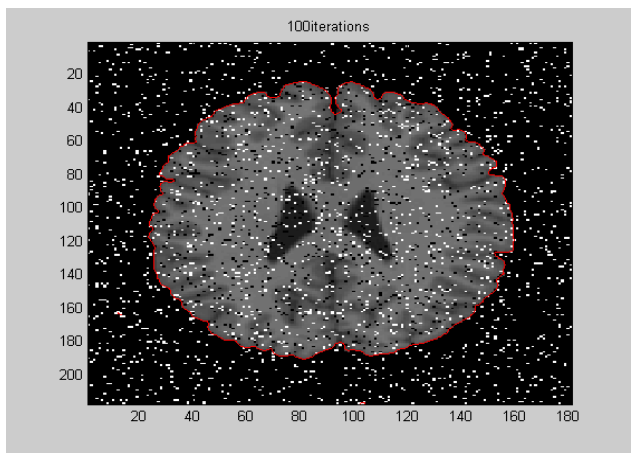

Figure (4b) the proposed model with 100 iterations 


\section{CONCLUSION}

In this paper, we proposed a region based segmentation of satellite images added with ten percentage of salt and pepper noise using active contours model and level set evolution. The results of this paper confirmed that the proposed method of global region based segmentation with active contours and level set evolution with SPF function showed perfect locked segmentation with lesser number of iterations and computational time. At the same time, the performance of image segmentation algorithms can be improved by modernization of classic velocity of active contours.

\section{REFERENCES}

[1] N. Xu, N. Ahuia, R. Bansal, Object segmentation using graph cuts based active contours, Computer Vision and Image Understanding 107 (2007) 210-224

[2] M. Kass, A. Witkin, D. Terzopoulos, Snakes: active contour models, International Journal of Computer Vision 1 (1988) 321-331.

[3] T. Chan, L. Vese, Active contours without edges, IEEE Transaction on Image Processing 10 (2) (2001) 266-277.

[4] V. Caselles, R. Kimmel, G. Sapiro, Geodesic active contours, in: Processing of IEEE International Conference on Computer Vision'95, Boston, MA, 1995, pp. 694-699.

[5] V. Caselles, R. Kimmel, G. Sapiro, Geodesic active contours, International Journal of Computer Vision 22 (1) (1997) 61-79.

[6] J. Lie, M. Lysaker, X.C. Tai, A binary level set model and some application to Munford-Shah image segmentation, IEEE Transaction on Image Processing 15 (2006) 1171-1181.

[7] D. Mumford, J. Shah, Optimal approximation by piecewise smooth function and associated variational problems, Communication on Pure and Applied Mathematics 42 (1989) 577-685.

[8] L.A. Vese, T.F. Chan, A multiphase level set framework for image segmentation using the Mumford-Shah model, International Journal of Computer Vision 50 (2002) 271-293.

[9] R. Ronfard, Region-based strategies for active contour models, International Journal of Computer Vision 46 (2002) 223-247.

[10] N. Paragios, R. Deriche, Geodesic active regions and level set methods for supervised texture segmentation, International Journal of Computer Vision 46 (2002) 223-247.

[11] A. Blake and M. Isard, Active Contours.. Cambridge, MA: Springer, N. Paragios and R. Deriche, "Geodesic active contours and level for the detection and tracking of moving objects," IEEE Trans. Pattern Anal. Mach. Intell., vol. 22, no. 3, pp 226-280, Mar. 2000.

[12] T. Zhang and D. Freedman, "Tracking objects using density matching and shape priors," in Proc. Int. Conf. Comput. is., 2004, pp

[13] N. Paragios, Y. Chen, and O. Faugeras, Handbook of Mathematial Models in Computer Vision.. New York: Springer, 2005.

[14] O. Michailovich, Y. Rathi, and A. Tannenbaum, "Image segmentation using active contours driven by the bhattacharyya gradient flow," IEEE Trans. Image Process., vol. 15, no. 11, pp. 2787-2801, Nov. 2007.

[15] V. Caselles, R. Kimmel, and G. Sapiro, "Geodesic active contours," Int. Comput. Vis., vol. 22, no. 1, pp. 61-79, Feb. 1997.

[16] C.Y. Xu, A. Yezzi Jr., J.L. Prince, On the relationship between parametric and geometric active contours, in: Processing of 34th Asilomar Conference on Signals Systems and Computers, 2000, pp. 483-489

[17] S. Osher, R. Fedkiw, Level Set Methods and Dynamic Implicit Surfaces, Springer-Verlag, New York, 2002.
[18] Tsai, A. Yezzi, A.S. Willsky, Curve evolution plementation of the Mumford-Shah functional for image segmentation, denoising, interpolation, and magnification, IEEE Transaction on Image Processing 10 (2001) 1169- 1186

[19] C.M. Li, C. Kao, J. Gore, Z. Ding, Implicit active contours driven by local binary fitting energy, in: IEEE Conference on Computer Vision and Pattern Recognition, 2007

[20] Li,C. Kao, J.C. Gore, Z. Ding, Minimization of region-scalable fitting energy for image segmentation, IEEE Transaction on Image Processing 17 (2008) 1940-1949.

[21] Shi W.C Karl, Real-time tracking using level sets, IEEE Conference on Computer Vision and Pattern Recognition 2 (2005) 34-41.

[22] P. Perona, J. Malik, Scale-space and edge detection using anisotropic diffusion, IEEE Transaction on Pattern Analysis and Machine Intelligence 12 (1990) 629-640.

Ganta Raghotham Reddy, received his Bachelor of engineering degree in Electronics \& Communications from Gulbarga University, $\mathrm{He}$ obtained his M.Tech in Digital Communication Engineering from Maulana Azad National Institute of Technology (MANIT), Bhopal. Currently an Assistant Professor in the department of Electronics and Communication Engineering, Kakatiya Institute of Technology and Science (KITS), Warangal, He is a Research Scholar pursuing PhD at Osmania University College of Engineering, Osmania University, Hyderabad. Life member of IETE, ISTE.

Kama Ramudu, received his Bachelor of engineering degree in Electronics \& Communications from V.R.Sidhartha Engineering College,vijayawada Nagarjuna University,Guntur. He obtained his M.Tech in Digital Communication Engineering from Kakatiya Institute Of Technology and Science,Kakatiya University, Warangal Currently an Assistant Professor in the department of Electronics and Communication Engineering, Kakatiya Institute of Technology and Science (KITS), Warangal. member of IETE.

Azmeera Srinivas, received his M.Tech, degree in Digital communication Engineering from Kakatiya Institute of Technology and Science, Kakatiya University, Warangal. He is the university topper in his M.Tech, KITS, Warangal and also received B.Tech in Electronics and communication Engineering from KITS(S),Huzurabad,Karimnagar, JNTUH. Currently an Assistant Professor in the department of Electronics and Communication Engineering, Kakatiya Institute of Technology and Science (KITS), Warangal. member of IETE.

R Rameshwar Rao, obtained his Bachelor of Engineering in Electronics and Communications from University College of Engineering, Osmania University, Hyderabad. He obtained both his M.Tech in Communication Engineering and Ph.d from IIT, Bombay. Currently a Professor in the Department of Electronics \& Communication Engineering and is Dean, Faculty of Engineering;, University College of Engineering Osmania University. Hyderabad. 